\title{
Darío y el modernismo en El Iris (1894) de Clemente Palma
}

\begin{abstract}
$\mathbf{A}^{\mathrm{L} \text { tratar de la época modernista peruana, conviene tener presente dos }}$ A hechos, afirma Luis Monguió: "Primero, en el Perú el modernismo aparece más tardíamente que en el resto de América... Segundo, y debido quizás en parte a esa misma introducción tardía e importada, el modernismo que en toda Hispanoamérica fue ya de sí un amplio y multifacético movimiento, en el Perú se caracteriza por una menor rigidez en la, ruptura con el pasado". ${ }^{1}$
\end{abstract}

Esto no quiere decir, por supuesto, que en el Perú no hubiera temprana conciencia estética del nuevo movimiento, de su significado, y de las letras europeas, especialmente de las francesas. En relación con esto, hay que recordar que la segunda vez (la primera fue en la Revista de Artes y Letras, $\mathbf{1 8 8 8}$, de Chile) que usó Datío la palabra modernismo fue en un artículo titulado "Fotograbado", que vio la luz en El Perú Ilustrado (8 de noviembre, I890).* Consigna Allen W. Phillips que en este artículo Darío se refiere "con más precisión al nuevo espíritu revolucionario y la palabra modemismo se va llenando de significado concreto". ${ }^{2}$

I. Véase: La poesía posmodemista peruana, 1954, pp. 10-11.

* [Por primera vez el "Fotograbado" de Ricardo Palma, en el Diario de Centro-América, Guatemala, 23 de agosto de 1890, según Evelyn Uhrham Irving, "Rubén Dario's First Days in Guatemala", en Hispania, mayo de 1963, XLVI, No 2, p. 321]

2 En "Rubén Daí́o y sus juicios sobre el modernismo", Revista Iberoameri. cana, Vol. XXIV, $\mathrm{N}^{\circ} 47$ (enero-junio, 1959), p. 42. Monguió observa referente a este asunto: "En efecto, según Estuardo Núñez, Rubén Dario fue presentado al Perú por doña Clorinda Matto de Turner en El Perú Ilustrado en 1890, y sólo a partir de 1893, a través de El Perí Artístico, se generalizó el conocimiento de su poesía y se difundió su concepto del modernismo". (OP. cit., p. 10). Véase Estuardo Núñez, "Las generaciones post-románticas de! Perú", Letras (Lima), N 5 (3er. cuatrimestre, 1936), p. 417. Para el origen y la evolución del término "modernismo", véase Max Henríquez Ureña, "Historia de un nombre", Breve 
En El Archivo de Rubén Dario de Alberto Ghiraldo se encuentran cuatro cartas de Ricardo Palma al poeta. En la primera, fechada marzo I2 de I894, se dice:

Mi querido amigo: Sin ninguna de usted a que dar respuesta, 'le remito un artículo de mi hijo Clemente, muchacho de 21 años y estudiante de jurisprudencia en esta universidad. Como verá usted las doctrinas literarias de Clemente están en oposición con las mias, que son para usted conocidas de antiguo.

El artículo de Manuel de la Cruz, que usted me envió en recorte y que hice reproducir en un diario de Lima, ha dado pretexto a mi retoño para hilvanar esa filípica. Allá el muchacho, que yo no he de imponerle mis ideas. 3

En la carta segunda, fechada el $I^{\circ}$ de mayo de I894, se dice:

Querido amigo: ¿Me ha olvidado usted? ¿Por qué no me escribe?

$\mathrm{Ha}$ dos meses le remití un artículo de mi hijo Clemente, contestando a Manuel de la Cruz, sobrie la cuestión del estilo. Le recomendaba que lo diese a conocer en la prensa bonaerense. Hoy le incluyo otro de crítica literaria por el mismo autorcillo, mozo que en breve cumplirá 22 años. Sus doctrinas literarias son, en mucho, opuestas a las mías. El muchacho es modernista, $y$, por consiguiente, entusiasta amigo de usted.

No sabemos si Rubén consiguió cumplir cabalmente con esta solícita iniciativa paterna ${ }^{4}$ de don Ricardo pero, sí, sabemos, leyendo la carta tercera (noviembre 30 de I894) del padre al poeta, que éste había enviado a Lima "varios números del Tiempo y tres de la Américas rotulados a Clemente. Merci bien".

bistoria del nodernismo, 1962, pp. 158-172. Véase también "Reflexiones en torno a la definición del Modernismo" por Ivan A. Schulman, en Cuadernos Americanos, Vol. CXLVII, julio-agosto de 1966, pp. 211-240.

3 Op. cit. (Santiago de Chile, 1940), pp. 140-141. En esta misma obra se refieren las circunstancias curiosas en que Darío conoció personalmente a Palma, en 1889. Véanse las páginas 97-101 de El arcbivo de Rubén Darío.

4 Ricardo Palma habla en la cuarta carta (1896) de la muerte de Manuel de la Cruz en estos términos: "Habrá usted lamentado, como yo, la muerte de Manuel de la Cruz, nuestro amigo de La Habana. Fse joven era un gran cerebro y un gran corazón. Fue una víctima más inmolada en el altar de la libertad antillana. Flor tropical, la agostó el invierno glacial de Nueva York" (pp. 107-108).

5 Debe tratarse de la Revista de América que publicaron Darío y Jaimes Freyre en la Argentina entre agosto y octubre de 1894, publicación muy rara que consta de tres números. Para el análisis de su contenido, véase Rafael Alberto Arrieta, La bistoria de la literatura argentina, tomo III, pp. 449-450; para la historia de la revista, véase Boyd $G$. Carter, "Dario periodista y redactor: En busca de la Revisia de América", Educación (Managua), N 18 (1961), pp. 40-50; - "Archivo Rubén Darío", Educación. N $\mathrm{N}^{\circ} 26$ (1954), pp. 49-52; _- "La 'Revista de América' de Darío y Jaimes Freyre" en Revista Mexicana de Cultura, Suplemento Dominical de El Nacional (México), No 1018 (octubre 2, 1966), pp. 1-2. 
Unas once semanas antes de publicarse en Buenos Aires el primer número de la Revista de América (I9 de agosto de 1894), y unas tres semanas después de aparecido el primer número de la Revista Azul (6 de mayo de 1894) de Gutiérrez Nájera y de Díaz Dufóo en México, apareció en Lima el primer número de El Iris (2* época, ${ }^{\circ}$ de junio de r894). En un aviso firmado por Vicente $\mathbf{H}$. Delgado, ${ }^{6}$ el antiguo director, se dice: "No siéndome posible continuar en la dirección de este periódico, se ha hecho cargo de ella el conocido escritor señor Clemente Palma".

El Iris ( 1894$),{ }^{7}$ revista mensual de literatura y ciencias, hoy publicación tan rara como apreciada por los coleccionistas conocedores del asunto, debe considerarse como el primer órgano del modernismo ${ }^{3}$ en el Perú. A su director, Clemente Palma, a la sazón de apenas 22 años de edad, le cupo ser el mayor animador de revistas literarias peruanas al servicio de la nueva literatura. Además de El Iris, fue el director de los

6 No hemos visto ningún número de El Iris publicado bajo la dirección de Delgado. En cuanto a sus ideas sobre la literatura insiste en "la necesidad de la educación artística; he aquí la necesidad de estudiar a los clásicos". Observa que los que creen que no hay poesía sin versos se equivocan puesto que la poesía está en el fondo y no en la forma. "Por esta razón Rubén Darío ha dicho: 'Emilio Castelar es un enorme poeta que no rima"." Dice: "Trabajemos con calma, con gusto, corrigiéndonos cada vez: cincelemos una escultura. No es la fecundidad lo que hace vivir al artista, sino su más perfecta producción". Cita a Manuel González Prada: "Lo que poco cuesta, poco dura. Las obras que admiran y deleitan a la Humanidad han sido pensadas y escritas en horas de soledad y recogimiento, costando a sus autores el hierro de la sangre y el fósforo del cerebro". Quizás dirija Delgado estos sabios consejos especialmente a Chocano. En estos comentarios se siente el recuerdo de "El Atte" de Gautier, de la estética de Flaubert así como de la admonición de Boileau: "Un sonnet sans défaut vaut seul un long poème"; "Hâtezvous lentement, et, sans perdre courage / Vingt fois sur le métier remettez votre ouvrage". Véase "Borroneando" por V. H. Delgado en El Iris, $\mathrm{N}^{\circ} 4$ (septiembre de 1894), pp. 123-125.

7 Hemos estudiado los números 1-5 de El lris correspondicntes a los meses de junio, julio, agosto, septiembre, octubre; tatal 160 páginas, tamaño $24 \times 18$ centímetros. Leemos en el número cinco (p. 153); "Advertimos con tiempo a nuestros suscriptores que esta revista subirá el precio de la suscripción trimestral a un sol, pues nos proponemos hacer algunas mejoras en ella desde el mes de enero. Asimismo, avisamos que nuestro próximo número correstonderá a los meses de noviembre y diciembre con el cual terminará el tomo II del İris y el trimestre actual. Hacemos esto con el fin de comenzar con el año 1895 el tomo III". Al parecer no se publicaron sino cinco númercs de El Iris. Me escribe (carta fechada el 8 de noviembre de 1966) don Federico Schwab, de Lima: "De acuerdo çn el fichero de la Biblioteca Nacional el último número publicado es el No 5 del $1^{\circ}$ de octubre de 1894... He tomado el dato del Boletín de la Biblioteca Nacional, Año 2, No 6, p. 141, Lima, 1945".

B Englekirk asienta el juicio siguiente sobre esta revista: "De muy especial interés para un conocimiento de los comienzos del Modernismo en el Perí es el artículo de C. Palma sobre 'La decadencia en América" ". Véase "La literatura y la revista literaria en Hispanoamérica". Revista Iboroamericana, Vol. XXVIII. N ${ }^{0}$ 53 (enero-junio. 1962), p. 20. Es todo lo que dice acerca de ella. Max Henríquez Ureña no menciona El Iris en su Breve historia del modernismo. 
números $4^{\mathrm{T}-7 \mathrm{I}}$ de Prisma (1905-1907), la revista de más importancia literaria y cultural que se publicara en el Perú en los comienzos del presente siglo. Hay colaboraciones de Palma, hijo, en cada uno de los $7^{\mathrm{I}}$ números de que consta la colección completa de Prisma.

En la primera página del primer número de El' Iris se publica "Una Carta literaria" de Mercedes Cabello de Carbonera9 (I845-1909), en forma de diálogo literario con un amigo suyo sobre los decadentes y los parnasianos. Según opina el amigo interlocutor, es "el cindelamiento fraseológico... la síntesis de esas dos hermanas gemelas, que se llaman Decadentes y Parnasianos. Hacer que en literatura sea el colorido, lo mismo que en la pintura, el rasgo característico que decida la supremacía de un cuadro...". El amigo de la autora declara que este tipo de literatura está en oposición con el concepto que tiene él de lo que debería ser la literatura: "yo creo que la literatura no es diversión de gentes ociosas, sino labor de apóstoles, de soldados y reformadores". Además, observa que casi ninguno de los decadentes de aquende el Atlántico es "demócrata y republicano de buena ley... Y he aquí que jóvenes de estas repúblicas democráticas, encuéntranse compelidos por la fuerza de la moda, y se dan a cantar la suntuosidad de un palacio, la magestad de una reina, la altivez de una princesa, con más entusiasmo que si cantaran a nuestra joven Democracia o a nuestra hermosa Libertad". Luego, nuestro intelocutor cita la opinión de Max Nordau para quien los decadentes son unos degenerados, víctimas de "la locura moral, de la imbecilidad y la demencia más o menos caracterizadas". El amigo-interlocutor concluye su comentario afirmando que los peruanos deberían estudiar los clásicos en vez de entusiasmarse por innovaciones literarias de mala muerte. "Por fin amiga mía; me explico que Goncourt, Ernesto Hello, Mallarmé, Verlaine se declaren decadentes y parnasianos; a ellos los admito como la más alta y bella eflorescencia de la literatura francesa, pero a los decadentes americanos, y más aún a los peruanos, los condenara a penitencia en descargo de sus pecados".

Así la señora Mercedes Cabello de Carbonera confunde decadentes con parnasianos, elogia lo utilitario y didáctico, desprestigia el arte por el arte, tolera la nueva literatura en Francia, pero la condena en el Perú y acusa a dichos innovadores en arte de ser conservadores en política. En forma más amplia la autora desarrolla algunas de estas mismas ideas

9 E. Anderson Imbert en Historia de la literatura bispanoamericana (1954, p. 232), le dedica el párrafo siguiente: Mercedes Cabello de Carbonera (Perú: 1845. 1909). solitaria, radical, estudió a Zola y fue naturalista en algunas novelas de ciudad, cebándose en la corrupción y la ruina de las clases altas (Blanca Sol, Las consecuencias). 
sobre la literatura y sobre la sociedad en su interesante ensayo "La novela moderna" que aparece como folletín en cuatro de los cinco números de El Iris. Rechaza tanto el romanticismo como el naturalismo. Para ella Bal$z a c$, Stendhal y Flaubert son realistas y no naturalistas " $y$ éste es su mejor título" (p. 95). Extraña que una persona con ideas tan cuajadas en notmas antagónicas a lo nuevo en la literatura, afirme que "los que se llaman conservadores no son más que insensatos que pretenden hacer vivir cadáveres" (p. I60).

El número 2 de El Iris (julio Iํ de I894) reproduce del Comercio un artículo de Clemente Palma titulado "La decadencia en América", en el que éste consigna su opinión sobre la "Carta literaria" de la Sra. Cabello de Carbonera. En este artículo el autor anda a tientas, balanceándose, podría decirse, en la cuetda floja de una crítica de doble cara. Si por una parte, afirma: I) "yo no simpatizo mucho con la escuela decadente", que considera como "el último estertor del idealismo absoluto, la agonía del romanticismo que intenta reanimarse tomando un aspecto nuevo". (p. 3.3); si dice 2) que no entiende aquel ideal del decadentismo que "embriaga y produce diablos azules" (p. 35); si no cree 3) que por medio de dicho movimiento se consiga "elevar nuestro nivel literario" (p. 37), si expresa, decimos, todas estas opiniones por una parte, por otra dice: "Es evidente que si el estandarte de la escuela tiene por lema la belleza, la escuela tiene legítimo derecho de existir, tanto en Francia con el místico Verlaine, el fonético Ghil, el enfermo $\mathrm{Ma}$ llarmé, los locos Baudelaire y Maupassant, y el tránsfuga del naturalismo, Karl Huysman [sic], como en América con Rubén Darío, Casal, Ambrogi y López Penha. La razón suprema del arte es la belleza" (p. 34).

La señota Cabello de Carbonera hace decir al amigo-interlocutor: "No me hable usted de los decadentistas peruanos, que me dan ganas de estallar en una estrepitosa carcajada" (p. 3). Observa Clemente: "En el Perú, la decadencia es desde poco más de un año acá cuando ha empezado a tener sectarios en la juventud... Los más conocidos de nuestros decadentes son los tres Pepes. José Antonio Román, José Fiansón y José S. Chocano. El primero, muy hábil, ha dado en la manía de imitar a Rubén Darío, por quien tiene pasión"... (pp. 36-37).

Clemente concluye su artículo con un comentario de doble cara, en que vuelve a expresar su admiración por una estética a la que vacila en adherirse como adepto entusiasta. "El decadentismo es una forma transitoria muy bella, pero que tiene menos robustez que el realismo. El ideal, el ideal absoluto se va, señora, se va. Se lo lleva el espíritu del siglo a la cripta bizantina como a. un rey decrépito de Oriente magni- 
ficamente vestido. Se va como aquellos soles de invierno que suelen a veces'alegrar la tierra con sus caricias de luz, pero que débiles como un anciano no pueden fecundar a la naturaleza con un beso de fuego!...".

Algunos adeptos de la nueva literatura tienden todavía en 1894 a identificar el decadentismo con el modernismo. Otros tienen el modernismo por un movimiento de tipo y de sabor hispánico, distinto del decadentismo. Así Enrique A. Carrillo que al hablar de Salvador Rueda, dice: "Rueda pertenece a la legión modernista de que él es pontífice en España y Rubén Darío en América, y en que descuellan personalidades tan brillantes como las de Gómez Carrillo, Soto Hall, Facio, Gavidia, Gutiérrez Nájera, Arciniegas y Casal, el malogrado poeta de la musa triste. El comprende que el genio y la inspiración encerrados en los viejos moldes retóricos, se enmohecen, se echan a perder. Por eso él es también un demoledor, un partidario del 'socialismo literario', que dice mi amigo Chocano, muy dado a hacer frases sonoras".10

Pedro César Dominici considerall la novísima literatura como la expresión coetánea de la evolución que estaba en tren de efectuarse en la cultura literaria y artística. "Absurdos de los retrógrados — dice- que quieren permanecer estacionados. La humanidad avanza, y la literatura no puede quedarse enmohecida ante el empuje del movimiento universal". Entonces observa irónicamente que siempre han tenido que luchar los nuevos contra los valores consagrados. "Sin embargo, es necesario atacar el decadentismo, es necesario ridiculizar el modernismo; como se hizo con el romanticismo, con el naturalismo, con el realismo, como se hace con el parnasismo y con el impresionismo". Más adelante dice: "Los retrógrados tiemblan, los moralistas se estremecen ante la ola civilizadora que se les viene encima, y entonces gritan con chillidos estridentes de hembras asustadas: son degenerados, extravagantes, ipsuitas, neurosténicos, vesánicos, simbolistas, deslicuescentes, decadentes, etc...".

Dominici pasa lista a los integrantes y a los promotores del decadentismo en Europa y caracteriza a algunos de ellos en estos términos:

Wagner, el que ha convertido la armonía en filosofia, $y$ ha transformado la frase instrumental en lenguaje intelectual, el que ha logrado hipnotizar hásta la seducción, creando la melodía infinita: es el jefe del decadentismo musical, el Padre de la Música del Porvenir; hoy todos los maestros imitan al maravilloso decadente, y sin embargo, están de acuerdo en llamarlo degenerado.

10 Véase Salvador Rueda, No 2, julio 1 de 1894, p. 47.

11 Las citas que se dan a continuación están tomadas de su ensayo "El simbolismo decadente", $\mathrm{N}^{\circ} 3$, agosto 1 de 1894, pp. 86-90. 
Carlos Baudelaire, el sublime neurótico, es decadente, y es tal vez el que más influye en la literatura moderna, y el que ha sido más plagiado por sus amigos: Io mismo sucede con Paul Verlaine, el gran poeta; y con Ibsen, el célebre noruego que ha revolucionado el drama y roto los convencionalismos del arte escénico.

Mallarmé, Rollinat, Richepin, Huyssmans (sic), Arthur Rimbaud, Verhaeren, Charles Morice, Moeterlink (sic), Gabriel Martín, etc., son todos extravagantes, enfermos, decadentes, (p. 89).

Al parecer, para Dominici, el decadentismo en su perfil americano se llama modernismo. Aunque el escritor venezolano no distingue claramente entre los dos términos, los dos movimientos no son idénticos en cuanto al concepto que tiene de ellos. Que no lo son, en opinión suya, lo atestigua la aseveración de que "la decadencia americana, aunque derivada de la decadencia francesa, se diferencia en mucho de ésta: la decadencia francesa sabe a éter y a absintio, la decandencia americana huele a flores y a selvas; en la decadencia francesa predomina el pesimismo, en la decadencia americana todavía existen agonías de optimismo histérica (sic); allả hay más fondo, más sensualismo, más neurosis; aquí hay más color, más armonía, más tono de luz, más belleza; allá vive el cerebro en constante agitación, la lucha es más terrible; aquí se vive bajo un cielo azul, hay más disgregación de voluntad, más ritmo" (p. 87).

En otro lugar del mismo artículo, Dominici define con más precisión todavía lo que considera que son los fines estéticos y el material poético de los modernistas decadentes americanos:

...La frase es un derrochamiento de armonía y luz: podrían llamarse Armonistas y Coloristas; el refinamiento del detalle, los tonos de la rima poética, la cadencia, el color en el sonido despiertan la sensación y forman la belleza. El ideal de estos decadentes, es hacer sentir hipnotizar en la voluptuosidad del fraseo convertido en música; estudiar a la Naturaleza como la estudia el artista, haciendo variar un paisaje por el solo hecho de variar la Iuz que le da sombras; sublimar la estética, reuniendo a la literatura, la pintura y la música.

En el decadentismo todo es argumento: una flor marchita, un mástil roto, las risas del río, los quejidos de un armonium, todo ante la fuerza del detalle es un estudio. El lenguaje se enriquece, y las Academias están obligadas a abrir el compás de sus diccionarios, si quieren ser obedecidas.

Ese es el decadentismo americano: una gran riqueza de imaginación, esfuminado con los colores del cielo tropical, aromatizada con los tiernos suspiros de nuestro joven fauno; y allá en el fondo, la Idea, pálida, muy pálida, abrasada al símbolo, que está siempre rojo, muy rojo... (p. 88). 
Hacia el final de su artículo, en evidente paráfrasis de unos versos de la Marseillaise, dice: “ ¡Decadentes! Los sátiros se ruborizan, no importa. Adelante Batallón Sagrado".

Clemente Palma, así como Dominici, insiste sobre el papel que podría y que debería tener la Naturaleza (con $\mathrm{N}$ mayúscula) en la nueva poesía de Hispanoamérica. En su artículo, "Más sobre el estilo",12 que es esencialmente un ensayo de tipo nacionalista sobre la necesidad de crear una literatura cabalmente americana, cita un comentario que le hizo Núñez de Arce a Darío acerca de la naturaleza americana como fuente de poesía:

Refiere Rubén Darío, que en una visita que le hizo Núñez de Arce, éste le dijo: que era en América, donde, para la lengua española estaba reservada la gran Poesía de nuestra maravillosa naturaleza, que "todavía no había tenido cantor digno de ella". Poesía robusta y sana, rebosante de savia y de fuego. "Eso debéis hacer vosotros los poetas nuevos de América, inspiraros en las grandezas naturales del Nuevo Mundo, escribir versos, poemas que tengan el aliento de aquella tierra ubérrima, señalar un nuevo campo a las musas españolas. Nosotros, los peninsulares, no tenemos aquí sino los gloriosos recuerdos del pasado, los monumentos de piedra, la historia. Vosotros sois el porvenir". La opinión, favorable a la doctrina que sostenemos, de un español egregio es de gran valor en esta cuestión, porque los españoles son los enemigos naturales de la independencia intelectual de América (pp. 68.69).

Clemente se refiere otra vez a Darío en la sección "Bibliografía y Notas" de El Iris, en relación con una crítica de Clarín contra Arturo Ambrogi y la nueva literatura americana. "El crítico español Leopoldo Alas -observa Clemente- le ha zurrado en un artículo dedicado a Rubén Darío y a los decadentes americanos. Pero ¡bah! El caso que hace Arturo de los paliques". 13

Enrique A. Carrillo afirma en su "Carta abierta" 14 a Juan Antonio Solórzano que no es decadente. "No obstante, creo también - diceque el decadentismo es oportuno en la época actual y oportuno, no solamente en Europa, sino en América" (p. 99). Aun cuando afirma Carrillo que no pertenece a ninguna secta ni bandería estética no disimula su admiración por el modernismo y por Darío. Dice:

12 En el número 3, $1^{\circ}$ de agosto de 1894, pp. 65-71.

is Ibid., p. 91 .

14 En el número 4, septiembre de 1894, pp. 97-104. [Esta misma carta, fechada en Lima, a 2 de abril de 1894, se publicó primeramente en el Repertorio Salvadoreño, San Salvador, julio de 1894, tomo IX, No 4, pp. 454-463. El Reperterio Salvadoreño figura en la lista de canjes de El Iris; cf. la nota 16 de este trabajo]. 
Sin embargo, hay cierto sistema con el que mucho simpatizo, y es aquel al que se ha bautizado con el nombre de modemismo, y cuyos fines son, al decir de su más prestigioso apóstol en América, Rubén Darío, "Ia elevación y la demostración en la crítica, con la prohibición de que el maestro de escuela anodino y el pedagogo chascarrillero penetren en el templo del Arte; la libertad y el vuelo; el triunfo de lo bello sobre to preceptivo, en la prosa, y la novedad en la poesía; dar color y vida y aire y flexibilidad al antiguo verso que sufte de anquilosis apretado entre tomados moldes de hierro". He aquí, admirablemente expresada, la reforma que yo ansio. Yo deseo que esta hermosa y rica lengua castellana, que hoy sufre de anquilosis, encerrada en los libros académicos, donde no abandona su aire estirado, su tono campanudo, su olor a viejo, donde se enmohece y reseca, cobre nuevos bríos, reciba como un aliento de vida, y reconquiste, en fin, por otros modos la antigua y vigorosa gallardía del siglo de oro.

La abundancia de poetas de talento dudoso o sin ninguno, cuyas obras veían la luz en el Perú en aquel entonces, lleva a este mismo crítico a decir que en el país se necesitaba la sangrienta causticidad de "un crítico como Bobadilla o Valbuena". Si en Lima se conoce, admira e imita "a los autores modernos y so comete cada cuarteto mironiano que clama al cielo y cada alejandrino a lo Dario que hace temblar", en el resto de la República donde se creía todavía que los escritores más en boga eran "Zorrilla, Espronceda y Castelar, se escribe tan malísimamente que ya no cabe más". Dedica el resto de su artículo a Chocano.

No aparecen sino dos colaboraciones de Darío en El Iris, ambos cuentos: "El Dios bueno" (No 2, julio de 1894, pp. 49-52)** y "Pequeños poemas en prosa: Luz de luna" ( $\mathrm{N}^{\circ}$ 3, agosto de r894, pp. 8I82). Cada párrafo de este último cuento (o poema en prosa) lleva un número romano en la versión que se publicó en $E l$ Iris.***

En los cinco números de esta revista que hemos examinado se hallan 94 colaboraciones ( 60 poemas, 24 ensayos, Io cuentos) por 48 escritores distintos. A continuación se dan la nómina y la nacionalidad de los más conocidos entre ellos. Así: Argentina: Leopoldo Díaz; Colombia: Julio Florez, Abrahám Z. López Penha, L. Torres Abanderro; Cuba: Diego Vicente Tejera; Ecuador: Lastenia Larriva de Llona, Numa P. Llona, Leónidas Pallares Arteta; Guatemala: Máximo Soto Hall; Pancmá: Darío Herrera, Adolfo García; Nicaragua: Rubén Darío; Perú: Mercedes Ca-

** [Con el título de "El buen Dios", en el Diario de Centro-América, Guatemala, 14 de agosto de 1890; cf. Evelyn Uhrhan Irving, trabajo citado en el primer asterisco, p. 321$]$.

*** [En Artes y Letras, Buenos Aires, 17 de junio de 1894, año II, N*29; cf. Emilio Carilla, Una etapa decisiva de Dario. Madrid, Gredos, 1967, p. 21, Arte y Letras figura en la lista de canjes de El Irits; véase la nota 16, p. 291 ]. 
bello de Carbonera, Enrique A. Carrillo, Enrique Castro y Oyanguren, José S. Chocano, Vicente H. Delgado, José Fiansón, Manuel Goazález Prada, Enrique López Albújar, Domingo Martínez Luján, Clemente Palma, Ricardo Palma, José Antonio Román; El Salvador: Arturo A. Ambrogi, Francisco A. Gamboa, Francisco Gavidia, Luis Lagos y Lagos, Juan Antonio Solórzano; Venezuela: N. Bolet Peraza, Pedro César Dominici.

Clemente anuncia en el número cuatro de El Iris (p. I28) que pronto tendrá "el gusto de publicar trabajos de Manuel de la Cruz, escritor cubano, y del guatemalteco Enrique Gómez Carrillo". Ninguna colaboración de estos escritores aparece en el número 5 de la revista.

Así, en El Iris se encuentran los nombres o las colaboraciones de casi todos los representantes más destacados del modernismo de aquel tiempo. Sin embargo, si allí se hallan referencias a Gutiérrez Nájera, a Casal, a S. Díaz Mirón, a Gómez Carrillo, a Alberto Masferrer, hay que notar que en la revista faltan los nombres de Martí, de Silva, de Nervo, de Díaz Rodríguez, de Lugones.

Entre los temas por los cuales más se preocupan los colaboradores en $E l$ Iris destacan aquellos que se relacionan con el modernismo y el decadentismo, con el naturalismo de Zola, y con lo que debería ser el contenido y el estilo de la literatura americana, o el "americanismo literario". Allí se muestra también cierto interés por la literatura del Japón.

No puede menos de sorprender al estudioso de este asunto el mucho interés que en dicha revista se manifiesta por Zola y por el naturalismo. Esto lo atestiguan los artículos de Francisco Gavidia, "Idealismo-Realismo" (No I); de Arturo A. Ambrogi, "Zola parisiense" (No 3); de N. Bolet Peraza, "Lourdes" (Nos. 3, 4, 5); de Mercedes Cabello de Carbonera, "La Novela moderna" (Nos. I, 2, 3, 5).

En suma, ¿a qué conclusiones referentes a la vigencia del modernismo en el Perú, en 1894, conduce este análisis del contenido de El Iris? Según creemos, a las siguientes:

I) Los escritores peruanos de aquel tiempo tenían plena conciencia del nuevo movimiento literario a que designan con los términos, "nueva literatura", "nueva poesía" (p. 35); "nuevo movimiento literario", "fiebre moderna" (p. 37); "evolución nueva de la literatura moderna", "escuela decadente" (p. 33); "literatura neurópata" (p. 4); "legión modernista" (p. 47); "modernismo" (pp. 87; 99); "Salvador Rueda, uno de los ídolos de los que blasonan de modernistas" (p. 106); "novísimo movimiento". (p. I04). Si a la sazón algunos literatos tienden todavía a identificar el modernismo con lo que llaman el decaden- 
tismo, otros, la mayor parte tal vez, hacen una distinción entre los dos términos.

2) Todos los peruanos de quienes se publicaron trabajos en El Iris, parecen conocer la obra de Darío. Algunos de ellos no sólo la tienen en mucho sino que defienden la nueva estética - sobre todo la parte de ella en que se insiste en la necesidad de renovación estilística-, aun cuando se permiten criticar a sus imitadores en el Perú.

3) Acaso basándose en los procedimientos descriptivos de Chocano, Clemente Palma y otros optan por un modernismo de perfil americano inspirado en la naturaleza del Nuevo Mundo.

4) A juzgar por las referencias y las firmas en El Iris, el mapa geográfico del modernismo para los peruanos en 1894 , se limitaba a México, Centro América, Cuba, Venezuela, Colombia, Ecuador y Perú. ${ }^{15}$ Allí apenas se menciona a Chile. En cuanto a la región del Río de la Plata, sólo figura en la revista el nombre de Leopoldo Díaz, más bien rezagado del romanticismo que modernista, y alguno que otro título de revista argentina en la sección conducida por Clemente, "Bibliografía y Notas". ${ }^{16}$

5) El material crítico en El Iris parece superar en calidad intrínseca a los poemas y a los cuentos que aparecieron en la revista. Estos jóvenes peruanos que por entonces andaban por los veinte años de edad, estaban notablemente enterados de la expresión cultural de Francia. Tampoco ignoraban los ideales y los valores nacionales e hispanoamericanos. En verdad, fue una generación privilegiada por el talento intelectual y crítico.

6) Como vehículo literario El Iris fue para la generación de Chocano, de Clemente Palma, de Enrique López Albújar, de Enrique A.

15 Para la lista y la nacionalidad de los colaboradores en la Revista Azul (1894-1896) de Manuel Gutiérrez Nájera y de Carlos Díaz Dufóo, véase "La Revista Azul. La resurrección fallida: Revista Azul de Manuel Caballero", por Boyd G. Carter en Las revistas literarias de México, México, INBA, 19.63, pp. 70-75.

16. Entre las revistas que recibe $\mathrm{El}$ Iris en canje se mencionan Artes y Letras (Buenos Aires), El Lápiz, El Duende, La Nube (Panamá), Las Tres Américas (Nueva York), Cosmópolis (Caracas), El Pensamiento, La Semana Literaria, La Pluma, Repertorio Salvadoreño, La Evolución. (El Salvador), La Habana Elegante y El Figaro (Cuba); Revista Gris (Colombia), La Revista Azul. No está claro si esta última revista es la Revista Azzl de Gutiérrez Nájera y de Díaz Dufóo de México o si se trata de otra publicación del mismo título en Barranquilla, Colombia [dirigida por Abraham Z. López Penha, que comenzó a publicarse con anterioridad a la Revista Azul, de México, el mes de enero de 1894; en la Biblioteca Menéndez y Pelayo, de Santander, signatura $\mathrm{N}^{\circ} 5606$, pueden consultarse los números 3, 4, 7 y 9 de marzo, abril, julio y septiembre de 1894, en los cuales figuran colaboraciones de los firmantes de El Iris; López Penha figura, en cambio, entre los de El Iris, y a él se refiere Clemente Palma en el $N^{\circ} 2$, p. 34 ]. 
Carrillo, de José Fiansón, lo que habría de ser la revista Contemporáneos (r909) para la generación de Leónidas N. Yerovi, de E. Bustamante y Ballivián, de José M. Eguren, y de Abraham Valdelomar. Además, en torno a El Iris se agruparon los primeros defensores de la estética modernista en el Perú.

Uninersity of Missouri

Boyd G. Carter 